\title{
A Carrier-Frequency-Offset Resilient OFDMA Receiver Designed Through Machine Deep Learning
}

\author{
Ang Li, Yi Ma, Songyan Xue, Na Yi, and Rahim Tafazolli \\ Institute for Communication Systems (ICS) \\ University of Surrey, Guildford, England, GU2 7XH \\ Email: (ang.li, y.ma, songyan.xue,n.yi, r.tafazolli)@surrey.ac.uk
}

\begin{abstract}
The aim of this paper is to handle the multifrequency synchronization problem inherent in orthogonal frequency-division multiple access (OFDMA) uplink communications, where the carrier frequency offset (CFO) for each user may be different, and they can be hardly compensated at the receiver side. Our major contribution lies in the development of a novel OFDM receiver that is resilient to unknown random CFO thanks to the use of a CFO-compensator bank. Specifically, the whole CFO range is evenly divided into a set of sub-ranges, with each being supported by a dedicated CFO compensator. Given the optimization for CFO compensator a NP-hard problem, a machine deep-learning approach is proposed to yield a good sub-optimal solution. It is shown that the proposed receiver is able to offer inter-carrier interference free performance for OFDMA systems operating at a wide range of SNRs.
\end{abstract}

\section{INTRODUCTION}

In orthogonal frequency-division multiple access (OFDMA) uplink communications, every user to the receiver link may suffer carrier frequency offset (CFO), which introduces inter-carrier interference (ICI) as well as mutual interference between users. Concerning the CFO for every user to be random and independent, it is often hard to conduct frequency synchronization at the receiver side; and this is referred to the well-known multi-frequency synchronization problem [1].

Given the receiver-side knowledge of CFOs, frequency synchronization might be applied onto each individual user's signal, which is obtained through the use of filter-bank [2]. However, the filter-bank approach cannot capture the signal energy leaked into others' user band; and this is the main reason to cause imperfectness for the frequency synchronization. A possible way to enhance the filter-bank approach is through the combination of iterative multiuser detection and frequency synchronization [3]-[5]. The fundamental bottleneck lies in the demand for accurate $\mathrm{CFO}$ and multiuser channel estimation as well as the decoding accuracy.

It might also be possible to conduct frequency synchronization at the transmitter side through the use of CFO pre-compensation approach, which turns the multi-frequency synchronization problem into a much simpler single-frequency synchronization problem [6], [7]. This approach lies in the hypothesis of accurate
CFO knowledge at the transmitter side, which is however hardly the case in practice due to the feedback delay and CFO dynamics. Moreover, the feedback for multiuser CFOs introduces extra signalling overhead, which is not favourable for advanced wireless applications such as $5 \mathrm{G}$, IoT or Tactile Internet that are demanding for a short frame length [8].

In this paper, we introduce a novel OFDM receiver architecture to handle the multi-frequency synchronization problem. Basically, the proposed receiver employs a new component named CFO-compensator bank, which consists of a set of parallel CFO compensators, with each in charge of a certain range of CFO; and aggregately the $\mathrm{CFO}$-compensator bank covers the whole $\mathrm{CFO}$ range. More specifically, the CFO-compensator bank is able to operate on two modes corresponding to blind frequency synchronization or non-blind frequency synchronization, respectively. The former assumes no receiver-side knowledge of CFOs, and in this case the received signal is fed into every $\mathrm{CFO}$ compensator. The output of the CFO-compensator bank is a number of parallel streams, which are demodulated and decoded individually. The decoded bit-stream that passed CRC is considered to be frequency synchronized. It is worth highlighting that the blind synchronization approach involves computation redundancy, which are sometimes considerable for a multiuser receiver. Alternatively, the non-blind frequency synchronization approach can be employed to minimize the computational redundancy. Given the receiver-side knowledge of CFOs, a specific CFO-compensator instead of all is chosen to conduct the frequency synchronization. The CFO knowledge can be obtained by employing any existing pilot-assisted or non-pilot assisted CFO estimation algorithms (e.g. [2], [6], [9]-[13] and many others). Here, we highlight that the proposed CFO-compensator is not sensitive to the CFO estimation accuracy as long as the estimation error falls into an acceptable range (e.g. 0.05 or smaller in absolute bias for the normalized CFO). This is one of remarkable advantages of the proposed frequency synchronization approach.

The major challenge for the CFO-compensator bank design lies in the optimization for each CFO compen- 
sator, which is a NP-hard problem. In order to handle this problem, a machine deep learning approach is proposed to suggest a good sub-optimum solution. The CFO range for each compensator is also carefully determined based upon both analytical and simulation work. The performance of the proposed receiver is evaluated in both the additive white Gaussian noise (AWGN) and mobile fading channels. It is demonstrated that the proposed receiver is able to offer ICI-free performance for OFDMA systems operating at a wide range of SNRs (e.g. $\mathrm{SNR} \leq 25 \mathrm{~dB}$ in mobile fading channels).

\section{OFDMA UPLINK COMMUNICATION MODEL AND PROBLEM FORMULATION}

Consider an OFDMA system where a set of users transmit their signals to the uplink receiver. Assuming the timing mismatch between users to be smaller than the length of cyclic prefix $(\mathrm{CP})$, the received signal after $\mathrm{CP}$ removal can be expressed by the following matrix form [14]

$$
\mathbf{y}=\sum_{l=0}^{L-1} \boldsymbol{\Omega}_{l} \mathbf{H}_{l} \mathbf{x}_{l}+\mathbf{v}
$$

with the subscript $l$ denoting the user index, and $L$ the number of users. In addition, $\mathbf{x}_{l}$ is the post-IDFT signal block sent by the $l^{\text {th }}$ user with the size $(M) \times(1)(M$ : the IDFT size), $\mathbf{H}_{l}$ the circulant Toeplitz channel matrix between the $l^{\text {th }}$ user and the receiver, $\mathbf{v}$ the AWGN; and finally, $\boldsymbol{\Omega}_{l}$ is the $(M) \times(M)$ diagonal matrix with its diagonal corresponding to the CFO for the $l^{\text {th }}$ user. In detail, the diagonal of $\Omega_{l}$ can be expressed by the following vector form

$$
\begin{aligned}
& \boldsymbol{\Omega}_{l}=\operatorname{diag}\left(\boldsymbol{\omega}_{l}\right), \\
& \boldsymbol{\omega}_{l}=\Omega_{l} \exp \left(\left[1, \ldots, \frac{j 2 \pi \omega_{l}(M-1)}{M}\right]^{T}\right)
\end{aligned}
$$

where $\omega_{l}$ is the CFO normalized by the OFDM subcarrier spacing with $\omega_{l} \in[-0.5,0.5], \Omega_{l}$ the $l^{\text {th }}$ user's CFO-related phase shift due to the CP removal, and the superscript $[\cdot]^{T}$ stands for the matrix transpose.

Prior to the DFT operation at the receiver, the CFO term $\Omega_{l}$ should be removed; or otherwise the ICI arises from the DFT operation. Given the knowledge of $\boldsymbol{\Omega}_{l}, \forall l$, joint frequency synchronization and multiuser detection can be conducted by minimizing the following Euclidean distance

$$
\hat{\mathbf{x}}_{l}=\underset{\mathbf{x}_{l}}{\arg \min }\left\|\mathbf{y}-\sum_{l=0}^{L-1} \boldsymbol{\Omega}_{l} \mathbf{H}_{l} \mathbf{x}_{l}\right\|^{2}
$$

where $\|\cdot\|$ denotes the Euclidean norm. This maximumlikelihood approach is optimum, however it does not take advantage of the OFDM structure, and it's computational complexity scales exponentially with the block size $M$. It is also possible to employ the iterative frequency synchronization and multiuser detection algorithm to

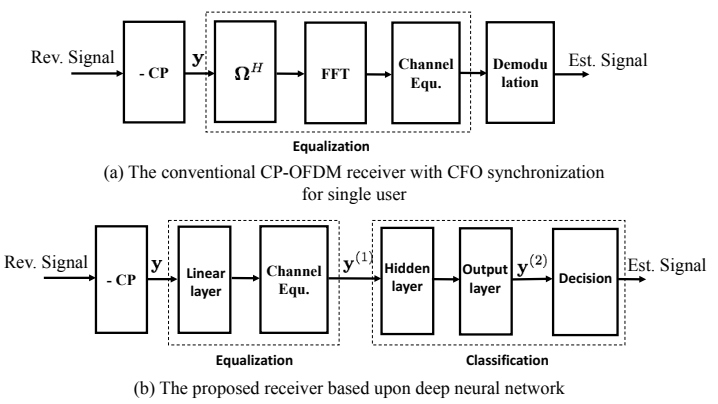

Fig. 1. Block diagram of the proposed OFDM receiver for multifrequency synchronization.

yield a sub-optimum solution [3], [4]. However, the computational complexity is still considerably high, and the frequency synchronization performance highly depends on the CFO and channel estimation accuracy.

The motivation of this work lies in our hypothesis of existing a (quasi-)linear frequency synchronization approach that can offer near-optimum multi-frequency synchronization performance for a wide range of SNRs. If this hypothesis gets supported, it is important to understand working conditions for the proposed approach.

\section{The Multi-Frequency Synchronization ALGORITHM AND ITS OPTIMIZATION THROUGH DEEP LEARNING}

\section{A. The Proposed Receiver Structure}

Fig. 1 (b) illustrates the block diagram of the proposed CP-OFDM receiver structure. To highlight the technical novelty, the block diagram of the conventional CPOFDM receiver is also shown in Fig. 1 (a). Mathematically, the proposed receiver can be described through two steps. The first step includes two linear-processing components with the key objective for the CFO and channel equalization. Given the Fourier decomposition $\mathbf{H}=\mathbf{F}^{H} \boldsymbol{\Lambda} \mathbf{F}$, where $\mathbf{F}$ denotes the $(M) \times(M)$ normalized DFT matrix, $\boldsymbol{\Lambda}$ the diagonal matrix consisting of the channel frequency response, and $[\cdot]^{H}$ the matrix Hermitian transpose, the first step can be expressed by the following matrix form

$$
\mathbf{y}^{(1)}=\boldsymbol{\Lambda}^{-1} \underbrace{\left(\mathbf{W}^{(1)} \mathbf{y}+\mathbf{b}^{(1)}\right)}_{\text {Linear Layer }}
$$

where the channel equalization component is simply the channel inverse $\Lambda^{-1}$, and the linear layer in (5) is responsible for the $\mathrm{CFO}$ compensation as well as the time-to-frequency domain transform. The term linear layer comes from the concept of neural network, where $\mathbf{W}^{(1)}$ is the weighting matrix of this layer, and $\mathbf{b}^{(1)}$ the bias vector.

The second step includes multiple nonlinear layers forming a neural network, which are responsible for the signal classification. Basically, the demodulation or 
decoding process is modeled by a signal classification process. An OFDM signal having $M$ subcarriers with each carrying a $Q$-state symbol can be classified into $N\left(=Q^{M}\right)$ bins, which can be represented by $J(=$ $\left.M \log _{2} Q\right)$ bits. Therefore, the output of the second step is a binary vector with the size of $(J) \times(1)$.

While the principle of the proposed CP-OFDM receiver will be introduced in Section III-B, it is worthwhile to highlight that the proposed receiver can be regarded as the generalized version of the conventional CP-OFDM receiver. The CFO and FFT component in the conventional CP-OFDM receiver is generalized by using the linear layer, and the demodulator is now replaced by the multi-layer neural network. More remarkably, every component in the CP-OFDM receiver is optimized individually. Thanks to the use of deep neural network (DNN) structure, every layer in the proposed receiver will be jointly optimized through the deep learning algorithm introduced in Section III-C. It will be shown that the joint optimization makes a big difference for the frequency synchronization.

\section{B. Principle of The DNN-based Receiver}

Concerning the linear layer in Fig. 1 (b) to be equivalent to a linear transform, we can represent the linear layer with

$$
\mathbf{W}^{(1)} \mathbf{y}+\mathbf{b}^{(1)}=\mathbf{\Gamma} \mathbf{y}
$$

where $\boldsymbol{\Gamma}$ is the linear transform matrix with the same size as $\mathbf{W}^{(1)}$. Then, the input to the signal classifier is

$$
\begin{aligned}
\mathbf{y}^{(1)} & =\boldsymbol{\Lambda}^{-1} \boldsymbol{\Gamma} \mathbf{y} \\
& =\boldsymbol{\Lambda}^{-1} \boldsymbol{\Theta} \mathbf{\Lambda} \mathbf{s}
\end{aligned}
$$

with

$$
\mathbf{s}=\mathbf{F} \mathbf{x} ; \boldsymbol{\Theta}=\boldsymbol{\Gamma} \boldsymbol{\Omega} \mathbf{F}^{H}
$$

Note that the user index $l$ is omitted as far as the single-user case is concerned, and we only consider the noiseless case for convenience. Moreover, the block $\mathbf{s}$ is the frequency-domain symbol block. Our interest is to understand: to what extend $\mathbf{s}$ can be determined given $\mathbf{y}^{(1)}$. This defines the feasibility condition for the classifier to work appropriately.

Basically, the DNN-based classifier is trained according to the maximum posterior probability. Assume $\mathbf{s}$ to be drawn from a finite set $\mathbf{A}=\left\{\mathbf{s}^{(1)}, \ldots, \mathbf{s}^{(N)}\right\}$. After the offline training, the DNN drops $\mathbf{y}^{(1)}$ into the bin labeled with $\mathbf{s}^{(n)}$ given

$$
\mathbf{s}^{(n)}=\underset{\mathbf{s}}{\arg \max } p\left(\mathbf{s} \mid \mathbf{y}^{(1)}\right)
$$

Define $\overline{\boldsymbol{\Theta}}=\boldsymbol{\Lambda}^{-1} \boldsymbol{\Theta} \boldsymbol{\Lambda}$ that varies randomly with respect to $\boldsymbol{\Lambda}$ and $\boldsymbol{\Omega}$. The classification error occurs when there exists an candidate $\mathbf{s}^{\left(n_{1}\right)}, n_{1} \neq n$, fulfilling

$$
\mathbf{y}^{(1)}=\overline{\boldsymbol{\Theta}}^{\left(n_{1}\right)} \mathbf{S}^{\left(n_{1}\right)}=\overline{\boldsymbol{\Theta}}^{(n)} \mathbf{S}^{(n)}
$$

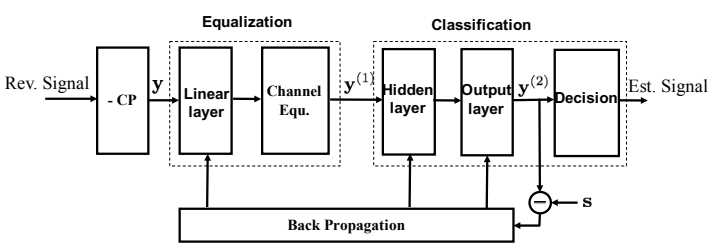

Fig. 2. Supervised deep learning for the receiver optimization.

The probability for this case must be minimized for the sake of the classification reliability; and this is the responsibility of the linear layer in the equalization component, with the objective of finding the matrix $\boldsymbol{\Gamma}$ that minimizes the probability. This task involves the linear programming problem, which is NP hard. Nevertheless, the DNN receiver can be trained through the supervised deep learning.

\section{Deep Learning for Receiver Optimization}

Fig. 2 illustrates the block diagram of the supervised deep learning for the DNN-based CP-OFDM receiver optimization. The DNN includes three layers: one linear layer, one hidden layer and one output layer, with their weighting matrices and bias vectors being updated through the stochastic gradient descent (SGD) algorithm with Adam optimizer at the learning rate $\alpha=0.001$, $\beta_{1}=0.9, \beta_{2}=0.999$ and $\epsilon=10^{-8}$ (see [15] for the detailed description of parameters). The activation function for the hidden layer is ReLU, and that for the output layer is Sigmoid. It is found that such configuration offers the best performance in computer simulations. Moreover, the channel equalization layer is simply the frequency-domain channel equalization with $\boldsymbol{\Lambda}^{-1}$, which varies with respect to the channel matrix $\mathbf{H}$. The input to the DNN is the CP-removed OFDM signal corrupted by the channel, random CFO as well as AWGN. The output is the $(J) \times(1)$ vector $\mathbf{y}^{(2)}$, with every $\left(\log _{2} Q\right)$ consecutive elements forming a group corresponding to a symbol in $\mathbf{s}$. The loss is measured by the categorical cross-entropy between $\mathbf{y}^{(2)}$ and $\mathbf{s}$. It is worthwhile to note that OFDM signals are complex, and they should be converted into real-equivalent signal in the DNN training procedure [16].

In the offline learning procedure, the DNN was trained for the CFO range $\omega \in\left(\omega^{(0)}, \omega^{(0)}+\delta\right)$, where $\delta(>0)$ is the bias from $\omega^{(0)}$. Section IV shows that the receiver performance generally degrades with the increase of $\delta$. However, there exists a threshold $\delta_{0}$, below which the performance degradation is not considerable. Inspired by this observation, we propose a CFO-compensator bank, which consists of a parallel set of CFO compensators, with each covering a certain range of $\mathrm{CFO}$; for instance, the first compensator covers $\omega \in\left[\omega^{(0)}, \omega^{(0)}+\delta\right)$, and the second covers $\omega \in\left[\omega^{(0)}+\delta, \omega^{(0)}+2 \delta\right)$, etc. Concerning there is only a frequency shift between CFO- 


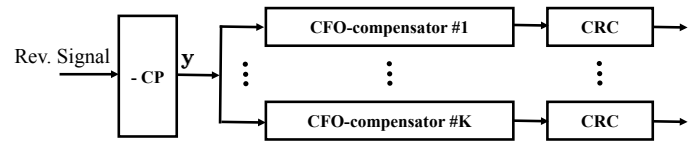

(a) Frequency synchronization without $\mathrm{CFO}$ knowledge

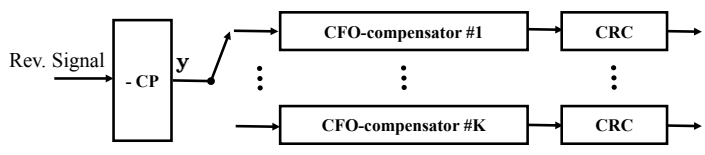

(b) Frequency synchronization with coarse $\mathrm{CFO}$ estimation

Fig. 3. Block diagram of the CFO-compensator bank.

compensators, the DNN training is only applied to the CFO compensator operating at $\omega \in\left[\omega^{(0)}, \omega^{(0)}+\delta\right)$, and all the others are just applied with a corresponding frequency shift.

Fig. 3 illustrates the block diagram of the proposed CFO-compensator bank. Basically, the CFOcompensator bank can operate in two modes. The first operating mode assumes no knowledge of the CFO. The received signal after $\mathrm{CP}$ removal is fed into all $\mathrm{CFO}$ compensators, with their output being further fed into the $\mathrm{CRC}$. The one passed CRC is accepted as the frequency synchronized result. In case there is no one passed CRC, a retransmission can be requested as usual. The second operating mode assumes a coarse CFO estimation, with the aim to select one or two appropriate $\mathrm{CFO}$ compensators for the frequency synchronization. The coarse CFO estimation does not need to be accurate. The estimation error can be roughly at the same level as $\delta$. Sometimes, the CFO estimation can just be simply based on users' mobility given sufficiently stable oscillators.

\section{Frequency Synchronization for OFDMA Uplink}

The proposed CP-OFDM receiver in Section III-C largely mitigates the multi-frequency synchronization problem in OFDMA uplink. Assuming: A1) all the uplink users have their $\mathrm{CFO}$ falling into the same range, i.e. $\omega_{l} \in\left[\omega^{(0)}+k \delta, \omega^{(0)}+(k+1) \delta\right), \forall l, k$, we can simply feed the received multiuser signal (1) into the proposed receiver shown in Fig. 3; as in this case, the proposed receiver renders the multi-frequency synchronization problem equivalently to the single-frequency synchronization problem.

It is worth noting that the assumption A1) holds when users within the same mobility range are grouped into the same frequency band using the network slicing, which is one of promising candidates for the $5 \mathrm{G}$ mobile technology [17]. Moreover, Section IV will show that the bias $\delta$ is large enough to support this assumption.

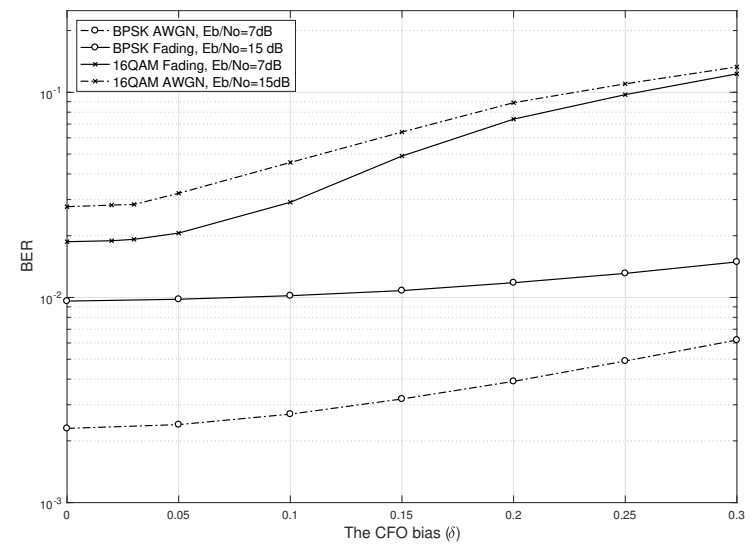

Fig. 4. BER vs. the CFO bias $(\delta)$ at the offline deep-learning stage.

\section{Simulation Results And Discussion}

The primary objective of our computer simulations is to evaluate the performance of the DNN-based CPOFDM receiver for both the offline training procedure and the online communication procedure. The OFDM system is configured by: $M=32$ subcarriers, the $\mathrm{CP}$ length of $8, L=1$ or 4 users with each having $(M) /(L)$ consecutive subcarriers. The modulation schemes are either BPSK or gray-coded 16-QAM, respectively. The communication channel is either AWGN or a 8-tap mobile fading channel with the power delay profile specified by 3GPP Pedestrian A [18]. Specifically, computer simulations are divided into the following three experiments.

Experiment 1: This experiment is mainly to conduct the supervised deep learning for the DNN-based CPOFDM receiver according to the setup specified in Section III-C and Fig. 2. The SNR at the deep-learning stage is: $\mathrm{Eb} / \mathrm{No}=7 \mathrm{~dB}$ for the AWGN channel, and 15 $\mathrm{dB}$ for the mobile fading channel; as this configuration is found to be the best for the deep learning.

Fig. 4 demonstrates the deep learning result (in terms of the bit error rate, BER) as a function of the $\mathrm{CFO}$ bias $\delta$ with the fixed configuration for $\mathrm{Eb} / \mathrm{No}$. It is observed that the performance generally degrades with the increase of $\delta$; this is what as expected since the linear layer is not able to synchronize a wide range of CFOs. The remarkable result appears within the range of $\delta \leq 0.05$, with which the performance degradation is not considerable. This interesting phenomenon motivates us to consider $\delta=0.05$ to be the best configuration for the CFO bias. This is because the configuration of $\delta<0.05$ results in the use of more CFO compensators without a considerable performance gain, and the configuration of $\delta>0.05$ results in considerable performance loss. Based upon this result, we divide the whole CFO range $(-0.5,0.5)$ into 20 sub-ranges to form the $\mathrm{CFO}$ - 


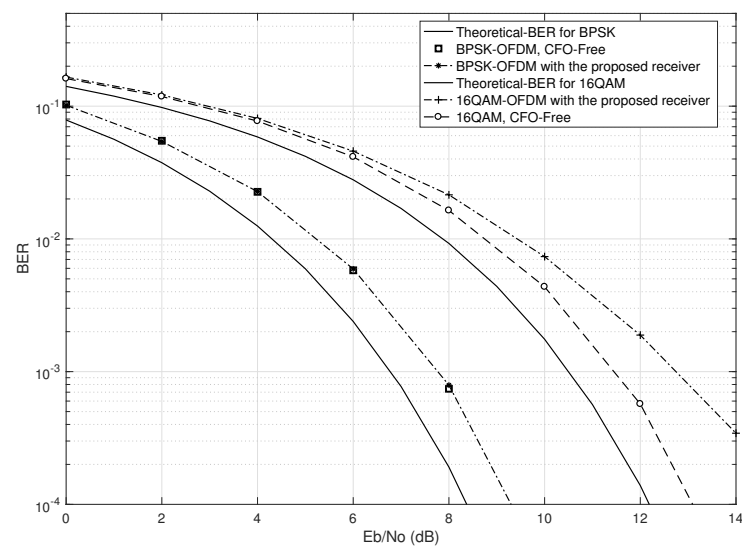

Fig. 5. BER for single-user OFDM $(L=1)$ as a function of Eb/No in the AWGN channel.

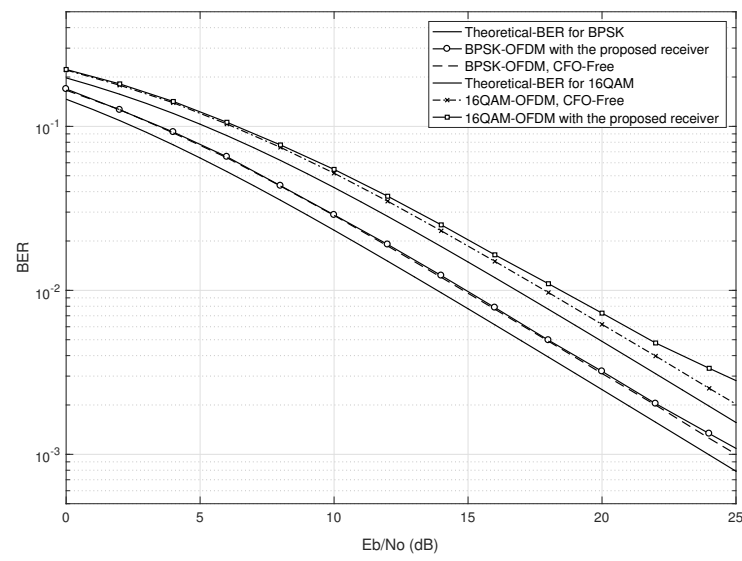

Fig. 6. BER for single-user OFDM $(L=1)$ as a function of Eb/No in the mobile fading channel.

compensator bank for the communication procedure in Experiment 2 \& 3.

Experiment 2: The objective of this experiment is to evaluate the DNN-based receiver in the single-user OFDM system. Throughout the simulation, we employ the receiver structure shown in Fig. 3 (b) for the frequency synchronization; the similar performance can be obtained for the receiver structure shown in Fig. 3 (a). The BER performance is obtained by averaging over sufficient Monte-Carlo trials. The BER performance for the AWGN channel is depicted in Fig. 5, and that for the mobile fading channel is depicted in Fig. 6. To facilitate our comparison, we also plot the theoretical-BER for BPSK/16QAM as well as the CFO-free CP-OFDM performance (considering the conventional CP-OFDM receiver) in both figures. Simulation results show:

1) There is a gap between the theoretical-BER for BPSK/16QAM and the BER for the CP-OFDM system. This gap is due to the power consumption for CPs.

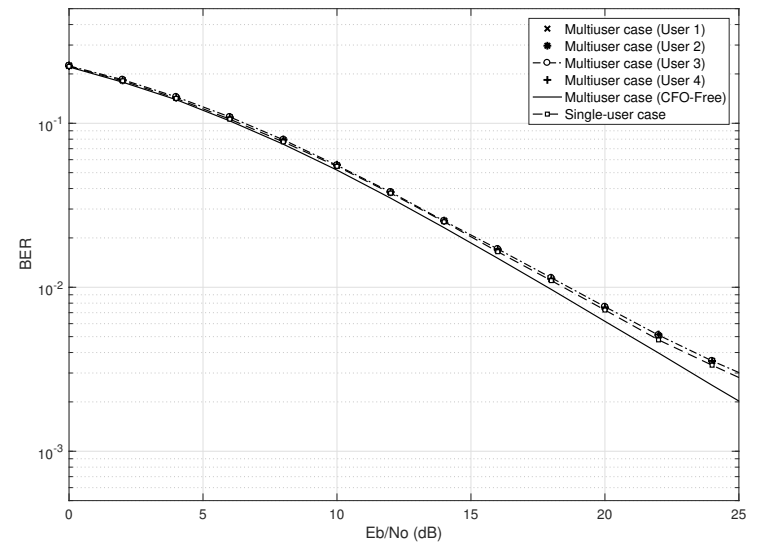

Fig. 7. BER for 16QAM-OFDMA $(L=4)$ as a function of Eb/No in the mobile fading channel.

2) For the BPSK-OFDM system, the proposed receiver can offer almost CFO-free performance throughout the whole SNR range both in the AWGN and mobile fading channels. There is only a very small performance gap (around $0.04 \mathrm{~dB}$ ) at high SNRs (e.g. Eb/No $=25 \mathrm{~dB}$ in the fading channel), which are however much higher than the typical SNR range for the BPSK-OFDM system.

3) The proposed receiver shows a bit worse performance when working for the 16QAM-OFDM system. In the AWGN channel, the almost CFO-free performance appears for $\mathrm{Eb} / \mathrm{No} \leq 6 \mathrm{~dB}$; and in the mobile fading channel, the performance is reasonably good for $\mathrm{Eb} / \mathrm{No} \leq$ $16 \mathrm{~dB}$. The major reason for having the seemingly worse performance lies in the use of $\mathrm{Eb} / \mathrm{No}$ for the performance evaluation. Given the same symbol energy to noise ratio (i.e. Es/No), Eb/No for 16-QAM is $6 \mathrm{~dB}$ lower than that for BPSK. In other words, the proposed receiver does not really get worse performance in terms of Es/No.

4) It is perhaps worth highlighting that the conventional pilot-assisted frequency synchronizer is very sensitive to the noise. Their CFO compensation performance is often poor at low SNRs due to the worse CFO estimation performance. Figs. 5-6 show that it is not the case for the proposed receiver. This is because the proposed receiver is designed in the way that is not sensitive to the CFO estimation error.

Experiment 3: The objective of this experiment is to evaluate the DNN-based receiver in the OFDMA system. The receiver is trained for the single-user system, and then straightforwardly applied into the OFDMA system $(L=4)$, where users have different random CFOs with the uniform distribution within the same CFO subrange. The BER performance for 16QAM-OFDMA over mobile fading channel is depicted in Fig. 7. It is observed that all users have identical BER performance throughout the whole SNR range. Moreover, the performance for OFDMA is almost the same as that for the single- 
user OFDM system; and there is only a very minor performance difference at high SNRs (around 0.01 dB or less at $\mathrm{Eb} / \mathrm{No}=25 \mathrm{~dB}$ ). This confirms our discussion in Section III-D.

\section{CONCLUSiON}

This paper has presented a novel DNN-based CPOFDM receiver to handle the multi-frequency synchronization problem inherent in OFDMA uplink communications. The proposed receiver was well optimized for both single-user and multiuser OFDM systems through the machine deep learning. Our computer simulations showed that the proposed receiver is able to offer almost CFO-free performance for both single-user OFDM and OFDMA systems operating at a wide range of SNRs without the need for accurate CFO estimation.

\section{ACKNOWLEDGMENT}

This work was partially funded by The $5 \mathrm{G}$ Innovation Centre (5GIC).

\section{REFERENCES}

[1] M. Morelli, C. C. J. Kuo, and M. O. Pun, "Synchronization techniques for orthogonal frequency division multiple access (OFDMA): A tutorial review," Proc. of the IEEE, vol. 95, no. 7, pp. 1394-1427, July 2007.

[2] J. J. van de Beek et al., "A time and frequency synchronization scheme for multiuser OFDM," IEEE J. Sel. Areas in Commun., vol. 17, no. 11, pp. 1900-1914, Nov 1999.

[3] M. O. Pun, M. Morelli, and C. C. J. Kuo, "Iterative detection and frequency synchronization for OFDMA uplink transmissions," IEEE Trans. Wireless Commun., vol. 6, no. 2, pp. 629-639, Feb 2007.

[4] M. Movahhedian, Y. Ma, and R. Tafazolli, "Blind CFO estimation for linearly precoded OFDMA uplink," IEEE Trans. Signal Process., vol. 58, no. 9, pp. 4698-4710, Sept 2010.

[5] M. Movahedian, Y. Ma, and R. Tafazolli, "Iterative carrier frequency offset estimation and compensation for OFDMA uplink," in IEEE 19th International Symposium on Personal, Indoor and Mobile Radio Communications (PIMRC), Sept 2008, pp. 1-5.
[6] M. Morelli, "Timing and frequency synchronization for the uplink of an OFDMA system," IEEE Trans. Commun., vol. 52, no. 2, pp. 296-306, Feb 2004.

[7] Y. Ma and R. Tafazolli, "Estimation of carrier frequency offset for multicarrier CDMA uplink," IEEE Trans. Signal Process., vol. 55, no. 6, pp. 2617-2627, June 2007.

[8] A. Ijaz, L. Zhang, M. Grau, A. Mohamed, S. Vural, A. U. Quddus, M. A. Imran, C. H. Foh, and R. Tafazolli, "Enabling massive IoT in 5G and beyond systems: PHY radio frame design considerations," IEEE Access, vol. 4, pp. 3322-3339, 2016.

[9] J. J. van de Beek, M. Sandell, and P. O. Borjesson, "ML estimation of time and frequency offset in OFDM systems," IEEE Trans. Signal Process., vol. 45, no. 7, pp. 1800-1805, Jul 1997.

[10] Y. Zeng and A. R. Leyman, "Pilot-based simplified ML and fast algorithm for frequency offset estimation in OFDMA uplink," IEEE Trans. Veh. Tech., vol. 57, no. 3, pp. 1723-1732, May 2008.

[11] S. Barbarossa, M. Pompili, and G. B. Giannakis, "Channelindependent synchronization of orthogonal frequency division multiple access systems," IEEE J. Sel. Areas in Commun., vol. 20, no. 2, pp. 474-486, Feb 2002.

[12] H. Bolcskei, "Blind estimation of symbol timing and carrier frequency offset in wireless OFDM systems," IEEE Trans. Commun., vol. 49, no. 6, pp. 988-999, Jun 2001.

[13] B. Park, H. Cheon, E. Ko, C. Kang, and D. Hong, "A blind OFDM synchronization algorithm based on cyclic correlation," IEEE Signal Process. Letts., vol. 11, no. 2, pp. 83-85, Feb 2004

[14] Y. Ma, N. Yi, and R. Tafazolli, "Channel estimation for PRP OFDM in slowly time-varying channel: first-order or secondorder statistics?" IEEE Signal Process. Letts, vol. 13, no. 3, pp. 129-132, March 2006.

[15] D. P. Kingma and J. Ba, "Adam: A method for stochastic optimization," CoRR, vol. abs/1412.6980, 2014.

[16] J. C. D. L. Ducoing, N. Yi, Y. Ma, and R. Tafazolli, "Using real constellations in fully- and over-loaded large MU-MIMO systems with simple detection," IEEE Wireless Commun. Letts., vol. 5, no. 1, pp. 92-95, Feb 2016.

[17] P. Rost, C. Mannweiler, D. S. Michalopoulos, C. Sartori, V. Sciancalepore, N. Sastry, O. Holland, S. Tayade, B. Han, D. Bega, D. Aziz, and H. Bakker, "Network slicing to enable scalability and flexibility in 5G mobile networks," IEEE Communications Magazine, vol. 55, no. 5, pp. 72-79, May 2017.

[18] 3GPP TS 36.101 v10.0.0, "LTE evolved universal terrestrial radio access (E-UTRA) "user equipment (UE) radio trasmission and reception",", Tech. Rep. 